MAGNETOHYDRODYNAMICS Vol. 54 (2018), No. 4, pp. 361-371

DOI: $10.22364 / \mathrm{mhd} .54 .4 .4$

\title{
SPECTRAL/HP ELEMENT MHD SOLVER
}

\author{
Alexander V. Proskurin ${ }^{1}$, Anatoly M. Sagalakov ${ }^{2}$ \\ 1 Altai State Technical University, 46 Lenin pr., Barnaul 656038, Russian Federation \\ ${ }^{2}$ Altai State University, 61 Lenin pr., Barnaul 656049, Russian Federation \\ e-Mail: k210@list.ru
}

A new MHD solver based on the Nektar++ spectral/hp element framework is presented in the paper. A velocity and electric potential quasi-static MHD model is used. The Hartmann flow in a plane duct and its stability, the Hartmann flow in a rectangular duct, and the stability of Hunt's flow are studied as examples. Exponential convergence is achieved and resulting numerical values have been found to have an accuracy up to $10^{-12}$ for state flows compared to an exact solution, and to $10^{-5}$ for stability eigenvalues compared to independent numerical results.

Introduction. It is well known that high-order methods have good computational properties, fast convergence, small errors, and the most compact data representation. To solve problems in hydrodynamics, high-order methods are necessary. The resolution of such problems involves the time-dependent simulation of transient flow regimes and the investigation of hydrodynamic stability. Of course, turbulent flows can be investigated using low accuracy schemes (achieving low precision results), but in most cases the problems of hydrodynamic stability in channels require the use of spectral methods. The classical Orr-Sommerfield equation has a small parameter $\mathrm{Re}^{-1}$ at the highest derivative which causes rapidly oscillating solutions. The first numerical calculation of eigenvalues for this equation [1] used a high-order finite difference scheme. Later, Orszag [2] obtained more accurate results and explained the convenience of using high-order methods for the solution of the problems of flow stability. A recent review of flow stability in complex geometries and the advantages and disadvantages of high-order methods can be found in $[3,4]$.

1. Problem formulation. Consider a flow of an incompressible, electrically conducting liquid exposed to a magnetic field. It is assumed that Rm $\ll$ Re. In this case, the magnetic field generated by the fluid motion does not affect the imposed magnetic field. This is correct for most engineering applications [5]. It is now possible to write the Navier-Stokes equation in the form

$$
\begin{aligned}
\frac{\partial \mathbf{v}}{\partial t}+(\mathbf{v} \nabla) \mathbf{v} & =-\frac{1}{\rho} \nabla p+\nu \Delta \mathbf{v}+\mathbf{F}(\mathbf{v}, \mathbf{H}), \\
\operatorname{div} \mathbf{v} & =0
\end{aligned}
$$

where $\mathbf{v}$ is the velocity, $p$ is the pressure, $\nu$ is the viscosity, $\rho$ is the density, $\mathbf{F}$ is the magnetic force, and $\mathbf{H}$ is the magnitude of the imposed magnetic field.

The Ohm's law reads as

$$
\mathbf{j}=\sigma(-\nabla \varphi+\mathbf{v} \times \mathbf{H})
$$

where $\mathbf{j}$ is the density of the electirc current, $\varphi$ is the electric potential, and $\sigma$ is the conductivity. Using the law of electric charge conservation ( $\operatorname{div} \mathbf{j}=0$ ), it is possible to derive the equation for the electric potential as

$$
\Delta \varphi=\nabla(\mathbf{v} \times \mathbf{H}) .
$$




\section{A.V.Proskurin, A.M. Sagalakov}

The system of equations (1) can be rewritten in the form:

$$
\begin{aligned}
\frac{\partial \mathbf{v}}{\partial t}+(\mathbf{v} \nabla) \mathbf{v} & =-\nabla p+\operatorname{Re}^{-1} \Delta \mathbf{v}+\operatorname{St}(-\nabla \varphi+\mathbf{v} \times \mathbf{H}) \times \mathbf{H}, \\
\operatorname{div} \mathbf{v} & =0, \\
\Delta \varphi & =\nabla(\mathbf{v} \times \mathbf{H}),
\end{aligned}
$$

where $\operatorname{Re}=L_{0} V_{0} / \nu$ is the Reynolds number, $\mathrm{St}=\sigma H_{0}^{2} L_{0} /\left(\rho V_{0}\right)$ is the magnetic interaction parameter (Stuart number), and $L_{0}, V_{0}$, and $H_{0}$ represent the scales of length, velocity and magnitude of the imposed magnetic field, respectively. The equation system (4) is also known as the MHD system in a quasi-static approximation in the form of electric potential. This system is widely used in theoretical investigations and accurately approximate many cases of liquid metal flows (for example, see the appropriate discussion and reference in [6]).

The boundary condition for the velocity at walls have the form:

$$
\mathbf{v}=0
$$

and the boundary condition for the electric potential at perfectly conducting walls is

$$
\varphi=\text { const. }
$$

The boundary condition for insulating walls is

$$
\frac{\partial \varphi}{\partial \mathbf{n}}=0 \text {. }
$$

2. Overview of numerical technique. The proposed new MHD solver has been developed on the basis of the open source spectral/hp element framework Nektar++ $[7,8]$. The incompressible Navier-Stokes solver (IncNavierStokesSolver) from the framework has been taken as the source for the MHD solver.

The IncNavierStokesSolver software uses the velocity correction scheme as described in $[8,9]$, assuming the time grid $t_{0}, t_{1}, \ldots, t_{n-1}, t_{n}, t_{n+1}$. Using a firstorder difference scheme, it is possible to define the intermediate velocity $\tilde{\mathbf{v}}$ from the equation:

$$
\frac{\tilde{\mathbf{v}}-\mathbf{v}_{n}}{\delta t}=-\left(\mathbf{v}_{n} \nabla\right) \mathbf{v}_{n}+\mathbf{F}_{n}+\mathrm{St} \cdot \mathbf{v}_{n} \times\left(-\nabla \varphi_{n}+\mathbf{v}_{n} \times \mathbf{H}\right),
$$

where $\mathbf{F}$ is a force acting of the fluid. At this stage, the Nektar++ software allows the force to be imposed, which acts on the flow. The electrical potential is found by solving the equation $\Delta \varphi_{n}=\nabla\left(\mathbf{v}_{n} \times \mathbf{H}\right)$ and after this the magnetic force in Eq. (8) is calculated. We derive the second intermediate velocity as

$$
\frac{\hat{\tilde{\mathbf{v}}}-\tilde{\mathbf{v}}}{\delta t}=-\nabla p_{n+1} .
$$

The Poisson equation

$$
\Delta p_{n+1}=\nabla\left(\frac{\tilde{\mathbf{v}}}{\delta t}\right)
$$

is immediately derived using $\operatorname{div} \hat{\tilde{\mathbf{v}}}=0$. Thus, at this stage, the divergence-free condition is approximately satisfied. Boundary conditions for the pressure are discussed in [9]. The last step of the velocity correction procedure is the equation:

$$
\left(\Delta-\frac{\operatorname{Re}}{\delta t}\right) \mathbf{v}_{n+1}=-\frac{\operatorname{Re}}{\delta t} \tilde{\mathbf{v}}+\operatorname{Re} \nabla p_{n+1},
$$

which allows us to find the next time-step velocity $\mathbf{v}_{n+1}$. The Nektar++ code can use the first, second and third order schemes.

362 


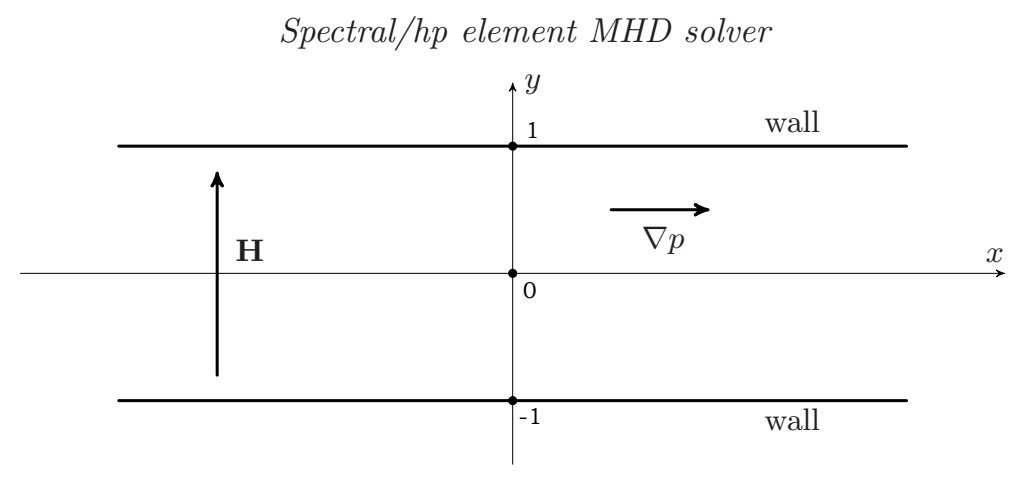

Fig. 1. The plane channel.

3. Hartmann flow in plane duct. Fig. 1 illustrates a flow in a plane duct. Two parallel infinite planes are installed at points $y= \pm 1$. The liquid between the planes flows under a constant pressure gradient $\nabla p$ in the $x$-direction. The magnetic field $\mathbf{H}$ is perpendicular to the planes. This is the Hartmann flow in the plane channel. According to [10], the solution of Eqs. (4) is

$$
\frac{u(y)}{u(0)}=\frac{\cosh (M)-\cosh (M y)}{\cosh (M)-1}
$$

where $M=\sqrt{\mathrm{St} \cdot \mathrm{Re}}, u(0)$ is the centreline velocity. Velocity graphs are shown in Fig. 2 for $M=10,100,10000$.

Fig. 3 displays a 2D mesh for numerical calculations of the flow. In Fig. 2, one can see large velocity gradients near the walls for the large $M$, and special attention should be paid to this part of the flow. It is possible to increase the accuracy by mesh concentration near the walls, where there are large velocity gradients. This is the $h$-type solution refinement. The high-order method can increase the accuracy by increasing the polynomials' order of the approximation, this is the $p$-refinement. For the flow calculations, we will combine both methods by using the order of approximation $p$ from 5 to 25 and by the mesh concentration near the walls with the coefficient $\beta$ ( $\beta=1$ for a uniform grid).

If assume the flow to be two-dimensional, all functions are independent on

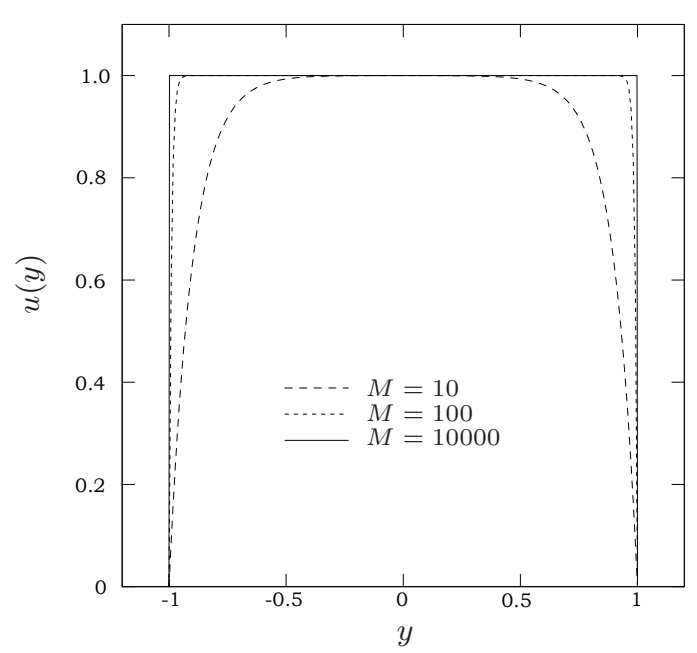

Fig. 2. Velocity of the Hartmann flow at different values of $M$. 


\section{A.V.Proskurin, A.M. Sagalakov}

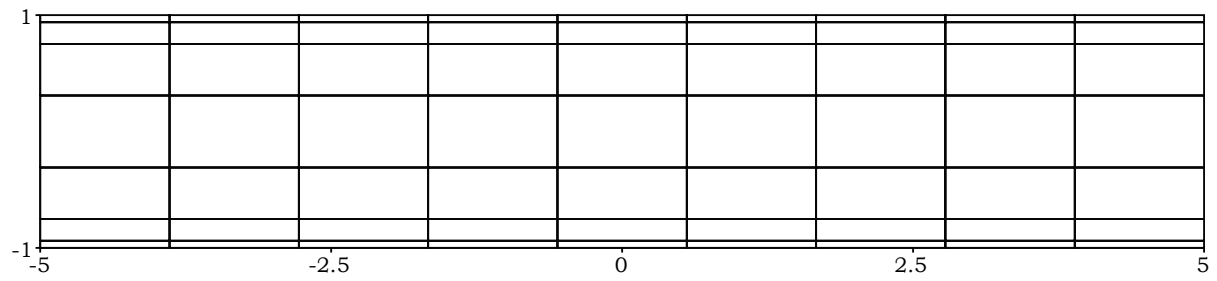

Fig. 3. A mesh to calculate the Hartmann flow, $\beta=0.05$.

the $z$-coordinate and $v_{z}=0$. This proposition leads to the equation $\Delta \varphi=0$; this means that boundary conditions for $\varphi$ are not needed. The boundary conditions for velocity and pressure are

$$
\begin{aligned}
\mathbf{v} & =0, \frac{\partial p}{\partial n}=0 \text { at walls, } \\
\frac{\partial \mathbf{v}}{\partial n} & =0, \frac{\partial p}{\partial n}=0 \text { at inflow and outflow. }
\end{aligned}
$$

In Table 1, maximum deviations from the exact solution are presented at $M=10 \sim 10^{4}$ for different orders of the polynomial approximation $p$. The state flow (Eq. 12) is calculated as a time-dependent flow with zero initial conditions. Table 1 includes the running time of the solver on the AMD Ryzen Threadripper 1920X machine with 12 threads.

4. Flow in rectangular duct. Let us consider a steady flow in a rectangular duct. A sketch of this flow is shown in Fig. 4. The rectangle in Fig. 4 is the cross-section of the channel and a uniform magnetic field is applied vertically. The liquid flows under a constant pressure gradient, perpendicular to the plane of the diagram. This flow was investigated analytically in $[11,12]$.

To compute the flow in rectangular duct, the authors use a mesh shown in Fig. 5. The Nektar++ software allows us to set up 3D problems, where the uniform direction is $z$, the number of Fourier modes is 2 (the minimum possible value).

The velocity convergence at the points $(0.95,0.0)$ for $M=10^{3}$ and $(0.98,0.0)$ for $M=10^{4}$ is presented in Table 2 for the case of perfectly electrically conducting walls. The table lists the mesh concentration coefficient $\beta$ and velocity values for

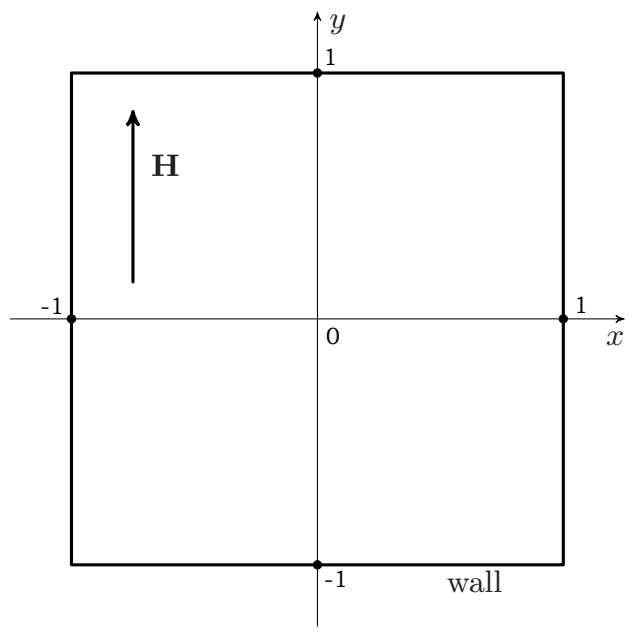

Fig. 4. The rectangular duct. 
Table 1. Maximum deviation from the exact solution (12), $M=10 \sim 10^{4}$.

\begin{tabular}{rllllllll}
\hline \hline$\beta$ & \multicolumn{2}{c}{0.5} & \multicolumn{2}{c}{0.05} & \multicolumn{2}{c}{0.005} & \multicolumn{2}{c}{0.0005} \\
$p$ & $M=10$ & $t, \mathrm{~m}: \mathrm{s}$ & $M=100$ & $t, \mathrm{~m}: \mathrm{s}$ & $M=10^{3}$ & $t, \mathrm{~m}: \mathrm{s}$ & $M=10^{4}$ & $t, \mathrm{~m}: \mathrm{s}$ \\
\hline 5 & 0.00035269 & $0: 00.50$ & 0.01206 & $0: 00.71$ & 0.0659132 & $0: 00.48$ & 0.116924 & $0: 01.29$ \\
10 & $8.55003 \cdot 10^{-10}$ & $0: 02.93$ & $3.67734 \cdot 10^{-6}$ & $0: 04.51$ & 0.000550293 & $0: 02.78$ & 0.00731443 & $0: 07.09$ \\
15 & $6.91253 \cdot 10^{-13}$ & $0: 21.02$ & $1.50558 \cdot 10^{-8}$ & $0: 16.32$ & $7.01068 \cdot 10^{-7}$ & $0: 12.65$ & 0.000215073 & $0: 23.43$ \\
20 & $7.76823 \cdot 10^{-13}$ & $0: 41.80$ & $4.32387 \cdot 10^{-11}$ & $0: 53.42$ & $9.98051 \cdot 10^{-10}$ & $0: 33.59$ & $4.2223 \cdot 10^{-5}$ & $0: 58.08$ \\
25 & $8.36775 \cdot 10^{-13}$ & $1: 39.52$ & $4.49063 \cdot 10^{-11}$ & $1: 58.71$ & $7.00766 \cdot 10^{-10}$ & $1: 15.66$ & $4.10316 \cdot 10^{-5}$ & $3: 36.74$ \\
\hline \hline
\end{tabular}

Table 2. Convergence of velocity values at the point $(x, y), M=10^{3}, 10^{4}$.

\begin{tabular}{|c|c|c|c|c|c|}
\hline \multirow[b]{2}{*}{$p$} & \multicolumn{2}{|c|}{$\beta=0.0005,(x, y)=(0.95,0.0)$} & \multicolumn{2}{|c|}{$\beta=0.0005,(x, y)=(0.95,0.0)$} & \multirow[b]{2}{*}{ memory usage, $\mathrm{Gb}$} \\
\hline & $M=10^{3}$ & $t, \mathrm{~m}: \mathrm{s}$ & $M=10^{4}$ & $t, \mathrm{~m}: \mathrm{s}$ & \\
\hline 5 & 1.197537688505880 & $0: 54.71$ & 1.105637945879100 & $2: 35.64$ & 0.041 \\
\hline 10 & 1.214652059488780 & $1: 31.85$ & 1.242371828471130 & $9: 29.99$ & 0.115 \\
\hline 15 & 1.212061289609800 & $5: 12.96$ & 1.234533071659520 & $24: 40.63$ & 0.358 \\
\hline 20 & 1.212043863156160 & $9: 30.71$ & 1.234851578015370 & 1h $12: 50$ & 0.962 \\
\hline 25 & 1.212044479951230 & $21: 53.03$ & 1.234834938798250 & $2 \mathrm{~h} 30: 03$ & 2.189 \\
\hline reference values & 1.21204510 & & 1.2348750 & & \\
\hline
\end{tabular}




\section{A.V.Proskurin, A.M. Sagalakov}

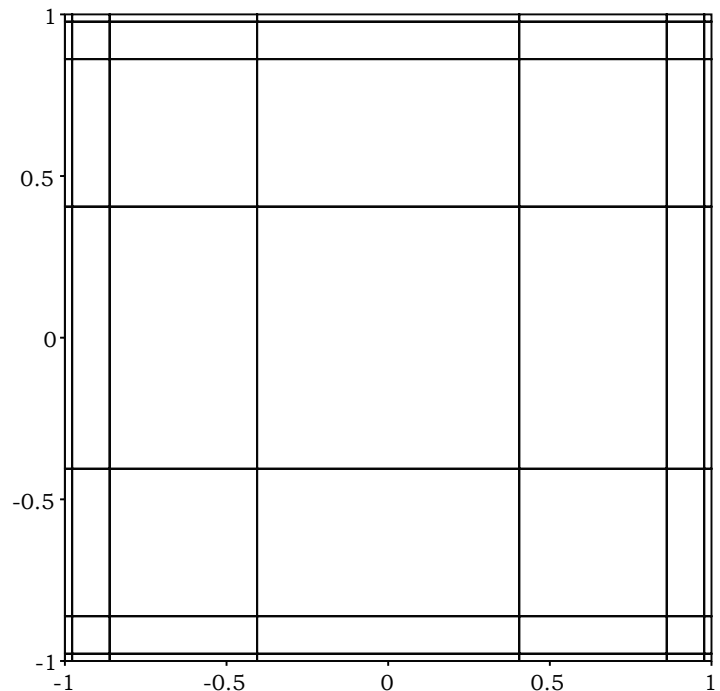

Fig. 5. A mesh to calculate the Hartmann flow in the duct, $\beta=0.01$.

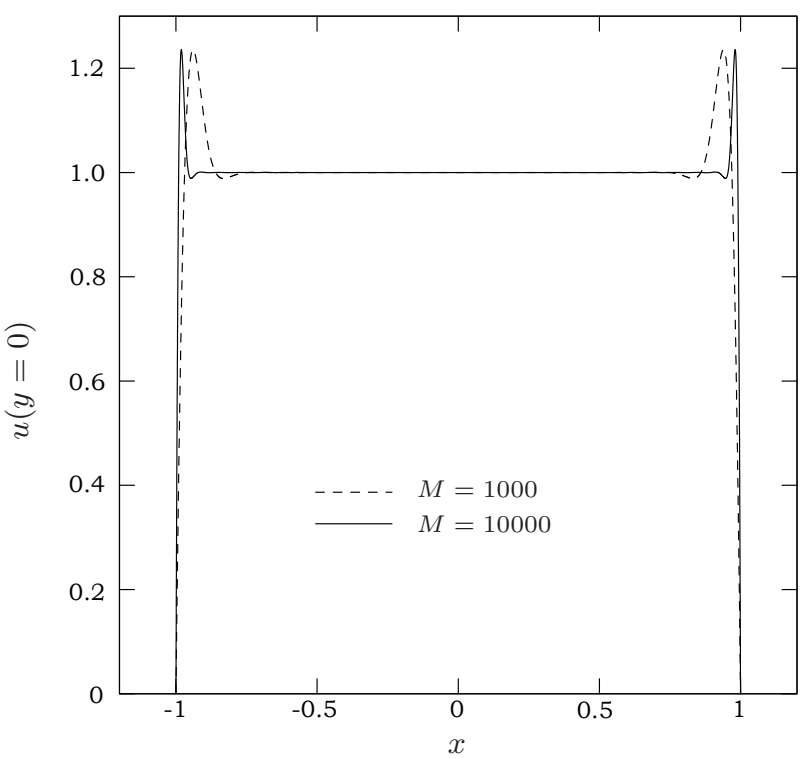

Fig. 6. Hartmann flow velocity in a rectangular duct at the line $y=0$ with ideal electrically conducting walls, $M=10^{3}, 10^{4}$.

different $p$ from 5 to 25, the time of calculation and memory usage for the AMD Ryzen Threadripper $1920 \mathrm{X}$ machine with 12 threads. The velocity for $M=10^{3}$, $M=10^{4}$ is shown graphically in Fig. 6 .

5. Stability problem. For a stability analysis, let us decompose the velocity, the pressure and the electric potential as

$$
\begin{aligned}
& \mathbf{v}=\mathbf{U}+\mathbf{v}, \\
& \varphi=\varphi_{0}+\varphi, \\
& p=p_{0}+p,
\end{aligned}
$$


where $U, \varphi_{0}$ and $p_{0}$ are the values of a steady-state flow, and $\mathbf{v}, \varphi$ and $p$ stand for small disturbances. The equation system (4) becomes linear:

$$
\begin{aligned}
\frac{\partial \mathbf{v}}{\partial t}+(\mathbf{U} \nabla) \mathbf{v}+(\mathbf{v} \nabla) \mathbf{U} & =-\nabla p+\operatorname{Re}^{-1} \Delta \mathbf{v}+\operatorname{St}(-\nabla \varphi+\mathbf{v} \times \mathbf{H}) \times \mathbf{H}, \\
\operatorname{div} \mathbf{v} & =0 \\
\Delta \varphi & =\nabla(\mathbf{v} \times \mathbf{H}) .
\end{aligned}
$$

From Eqs. (15), a linear operator A can be constructed:

$$
\mathbf{v}(x, y, z, T)=\mathbf{A}(T) \mathbf{v}(x, y, z, 0)=\lambda(T) \mathbf{v}(x, y, z, 0),
$$

where $T$ is the time interval. The linear operator $\mathbf{A}(T)$ is constructed numerically by a splitting procedure in the same way as in the case of nonlinear equations (4). In order to find an eigenvalue $\lambda(T)$, it is convenient to construct a Krylov subspace

$$
K_{n}\left(\mathbf{A}, \mathbf{v}_{0}\right)=\operatorname{span}\left\{\mathbf{v}_{0}, \mathbf{A}(T) \mathbf{v}_{0}, \mathbf{A}(T)^{2} \mathbf{v}_{0}, \ldots, \mathbf{A}(T)^{n-1} \mathbf{v}_{0}\right\},
$$

where $\mathbf{A}(T)^{i} v_{0}$ is obtained by direct calculation of

$$
\mathbf{v}_{1}=\mathbf{A}(T) \mathbf{v}_{0}, \mathbf{v}_{2}=\mathbf{A}(T) \mathbf{v}_{1}, \ldots
$$

Further, eigenvalues are calculated by standard numerical algebraic techniques, such as the Arnoldi method. The eigenvalues are obtained by Nektar++ in the form:

$$
\lambda(T)=m \cdot \mathrm{e}^{\theta i},
$$

and if $m>1$, the flow is unstable. The time-independent growth is $\sigma=\ln (m) / T$ and the time-independent frequency is $\omega=\theta / T$.

6. Hartmann flow stability. In Section 3, the Hartmann flow was considered. Now, we will investigate the stability of this flow. We take a Hartmann 2D flow disturbance (14) in the form:

$$
\{\mathbf{v}, \varphi, p\}=\mathbf{q}(x, y) \mathrm{e}^{i \alpha(x-C t)},
$$

where $\mathbf{q}(x, y)$ is the disturbance amplitude, $\alpha=2 \pi / \gamma$ is the wave-vector, $\gamma$ is the wavelength, $C=X+i Y$ is the disturbance phase velocity, $\alpha X=\omega$ is the frequency, $\alpha Y=\sigma$ is the growth of disturbance. When $\sigma \leq 0$, it means that the flow is stable.

The disturbance form (19) is widely used in hydrodynamics stability analysis and results in an eigenvalue problem similar to Eq. (16). As reference data, we take Takashima critical values [13] for 2D disturbances. In Table 3, the growths and frequencies of the disturbances are summarized for several cases. The values of Re and $M$ are taken from [13], and these are critical values of the Hartmann flow. The computational grid is shown in Fig. $3 ; n x$ and $n y$ are the number of cells in the horizontal and vertical directions. The length $L$ of the grid is set up by using a critical wave-vector $\alpha_{c}=2 \pi / L$. The boundary conditions at inlet and outlet are periodical. In Table 3, a complete coincidence is observed with the reference data from [13] and the convergence of the eigenvalues is achieved up to $10^{-7}$. Additionally, the table includes the time and memory usage (for the AMD Phenom FX-8150 processor with 8 threads). 
Table 3. Eigenvalues of the Hartmann flow.

\begin{tabular}{ccccccccc}
\hline \hline $\operatorname{Re}$ & $M$ & $\alpha$ & $n_{x} \times n_{y}$ & $p$ & $\sigma / \alpha$ & $\omega / \alpha$ & time & memory,GB \\
\hline 10016.2621 & 1 & 0.971828 & $6 \times 3$ & 10 & 0.233652452491593 & $4.14 \cdot 10^{-4}$ & $1 \mathrm{~h} 18: 12$ & 0.07 \\
- & - & - & - & 20 & 0.235517912202571 & $1.55 \cdot 10^{-6}$ & $3 \mathrm{~h} 28: 46$ & 0.33 \\
- & - & - & $8 \times 6$ & 10 & 0.235518158254341 & $-7.67 \cdot 10^{-7}$ & $1 \mathrm{~h} 47: 42$ & 0.11 \\
- & - & - & - & 15 & 0.235518939061233 & $8.97 \cdot 10^{-8}$ & $4 \mathrm{~h} 35: 36$ & 0.30 \\
- & - & - & - & 20 & 0.235518994976477 & $6.38 \cdot 10^{-8}$ & $11 \mathrm{~h} 03: 43$ & 0.77 \\
28603.639 & - & 0.927773 & $8 \times 6$ & 10 & 0.192137721274493 & $1.66 \cdot 10^{-6}$ & $3 \mathrm{~h} 19: 01$ & 0.13 \\
- & - & - & reference value & 0.192133 & & \\
65155.21 & 3 & 0.958249 & $8 \times 6 \quad 20$ & 0.169030377438432 & $1.16 \cdot 10^{-7}$ & $15 \mathrm{~h} 41: 21$ & 0.77 \\
- & - & - & from [13] & 0.169030 & & \\
\hline \hline
\end{tabular}

Table 4. Eigenvalues of the Hunt's flow, $M=10,10^{2}, 10^{3}$.

\begin{tabular}{|c|c|c|c|c|c|c|c|c|c|c|}
\hline$p$ & $\begin{array}{l}M=10 \\
\sigma / \alpha\end{array}$ & $\begin{array}{c}\beta_{x}=0.05, \beta_{y} \\
\omega / \alpha\end{array}$ & $\begin{array}{l}=0.1 \\
\text { time }\end{array}$ & $\begin{array}{l}M=10 \\
\sigma / \alpha \\
\end{array}$ & $\begin{array}{c}\beta_{x}=0.01, \beta_{y} \\
\omega / \alpha\end{array}$ & $\begin{array}{l}=0.5 \\
\text { time }\end{array}$ & $\begin{array}{l}M=10 \\
\sigma / \alpha\end{array}$ & $\begin{array}{c}\beta_{x}=0.01, \beta_{y} \\
\omega / \alpha\end{array}$ & $\begin{array}{l}=0.5 \\
\text { time }\end{array}$ & memory, Gb \\
\hline 5 & 0.7495498 & $-0.5494 \cdot 10^{-2}$ & 7:02 & 0.8109660 & -0.134660 & $5: 48$ & 0.4576533 & $2.41323 \cdot 10^{-2}$ & $2: 12$ & 0.042 \\
\hline 10 & 0.7579881 & $-0.3446 \cdot 10^{-3}$ & $15: 13$ & 0.4912104 & $-0.6873 \cdot 10^{-2}$ & $42: 59$ & 0.4799186 & $0.08964 \cdot 10^{-2}$ & $14: 12$ & 0.118 \\
\hline 15 & 0.7579733 & $-0.3348 \cdot 10^{-3}$ & 1h27:24 & 0.4907436 & $-0.8033 \cdot 10^{-2}$ & 1h50:50 & 0.4784210 & $0.14098 \cdot 10^{-2}$ & $2 \mathrm{~h} 21: 50$ & 0.365 \\
\hline 20 & 0.7579599 & $-0.3311 \cdot 10^{-3}$ & 5 h57:42 & 0.4907460 & $-0.8030 \cdot 10^{-2}$ & $2 \mathrm{~h} 42: 15$ & 0.4784201 & $0.14121 \cdot 10^{-2}$ & 3h50:34 & 0.972 \\
\hline from [15] & 0.7579413 & $-0.3337 \cdot 10^{-3}$ & & 0.4907415 & $-0.8028 \cdot 10^{-2}$ & & 0.5053902 & $0.14170 \cdot 10^{-2}$ & & \\
\hline
\end{tabular}




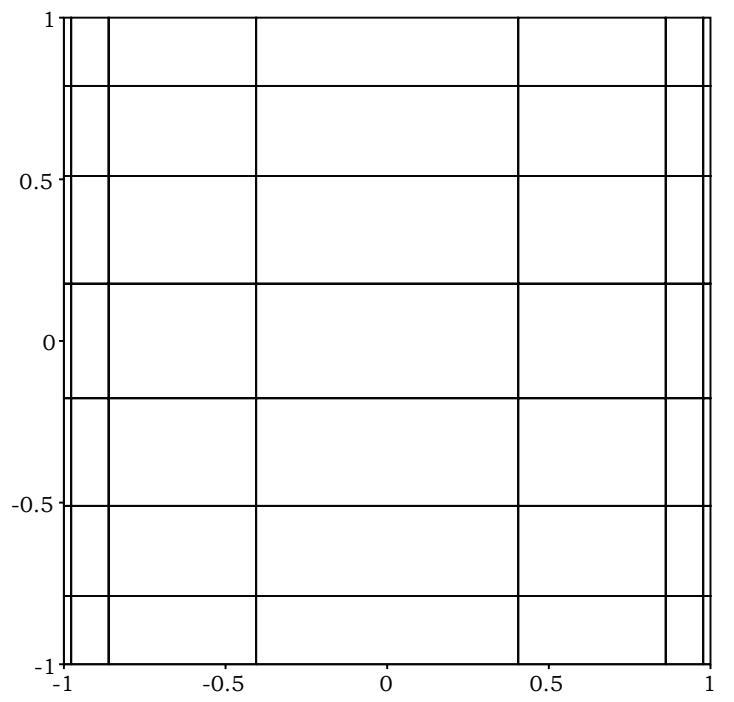

Fig. 7. A mesh to calculate the Hunt's flow, $\beta_{y}=0.5, \beta_{x}=0.01$.

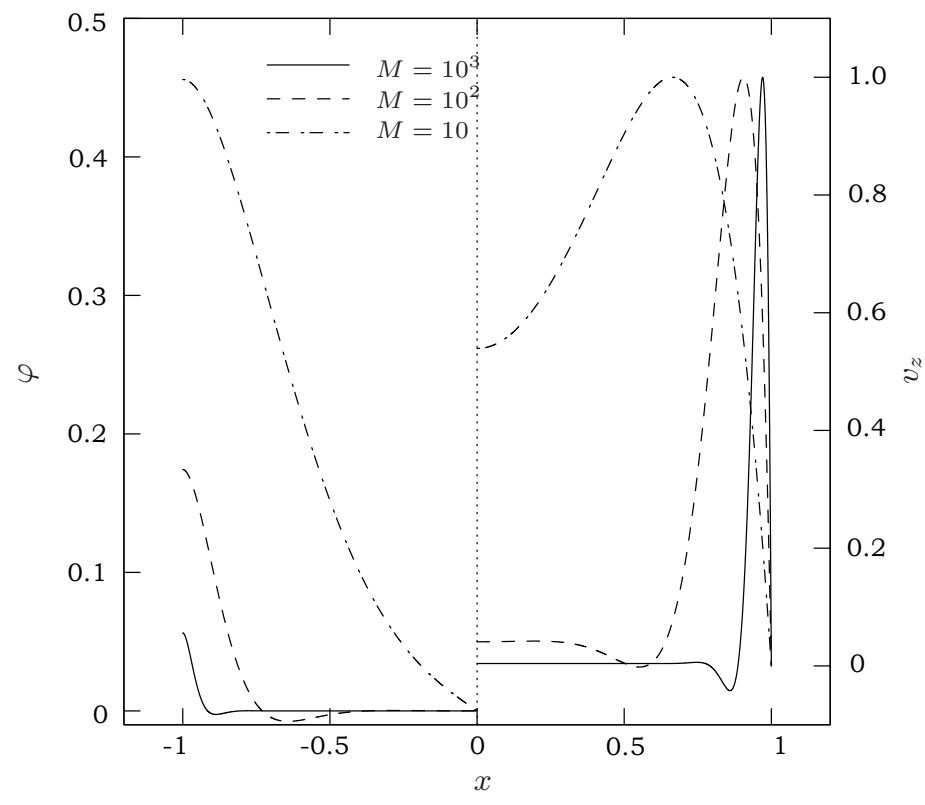

Fig. 8. Velocity $\mathbf{v}_{z}$ and electric potential $\varphi$ of Hunt's flow at the line $y=0, M=$ $10,10^{2}, 10^{3}$.

7. Stability of Hunt's flow. Consider a steady flow in a rectangular duct (Fig. 4), where the horizontal walls are perfectly electrically conducting and the vertical walls are perfectly electrically insulating. The flow was investigated in [14], and it is known since as the Hunt's flow. A mesh for the base flow and stability calculations is shown in Fig. 7. Fig. 8 displays a graph of the velocity over the line $y=0$ at $M=10,10^{2}, 10^{3}$. In Table 4 , the calculated eigenvalues are compared with the reference values from [15]; the time of calculation and the memory usage are presented. It is possible to see the numerical convergence by increasing the order $p$ and match with the reference values from [15] up to $10^{-5}$, excluding the case $M=10^{3}$. 


\section{A.V.Proskurin, A.M. Sagalakov}

Conclusion This paper presents the spectral/hp element solver for MHD problems based on the Nektar++ framework. The solver also makes it possible to investigate the stability of such flows. In order to demonstrate the solver's capacity, several examples were considered: the Hartmann flow in a plane duct and its stability, the Hartmann flow in a rectangular duct, and the stability of Hunt's flow. For the flows, it was easy to find steady-state solutions analytically, and these results were used as the reference test solutions. It was found that the margin of error decreases exponentially with the increasing degree of approximating polynomials, and an accuracy of $10^{-12}$ can be achieved. To estimate the costs of the computer time and memory, these data were listed in the tables for several cases. The computational costs for the stability calculations are large. One reason is that a non-stationary algorithm was used, which allowed to apply a non-stationary solver with small adaptations. To obtain eigenvalues with high accuracy, we should set a small time step. Another reason is that we considered the test examples in 2D for the Hartmann flow and in 3D for flows in the duct. Usually, these problems can be reduced to simpler cases described in $[13,15,16]$, which can be investigated with much lower computational costs. In this paper, the accuracy of the method is demonstrated using the well investigated examples. In general, our numerical technique is intended for complex geometry flows, where such simplifications are not possible.

\section{References}

[1] L.H. Thomas. The stability of plane Poiseuille flow. Physical Review, vol. 91 (1953), no. 4, p. 780

[2] S.A. Orszag. Accurate solution of the Orr-Sommerfeld stability equation. Journal of Fluid Mechanics, vol. 50 (1971), no. 4, pp. 689-703.

[3] L. Gonzalez, V. Theofilis, And S.J. Sherwin. High-order methods for the numerical solution of the BiGlobal linear stability eigenvalue problem in complex geometries. International Journal for Numerical Methods in Fluids, vol. 65 (2011), no. 8, pp. 923-952.

[4] V. TheofiLis. Global linear instability. Annual Review of Fluid Mechanics, vol. 43 (2011), pp. 319-352.

[5] D.Lee and H.Choi. Magnetohydrodynamic turbulent flow in a channel at low magnetic Reynolds number. Journal of Fluid Mechanics, vol. 439 (2001), pp. 367-394.

[6] D. Krasnov, O. Zikanov, and T. Boeck. Comparative study of finite difference approaches in simulation of magnetohydrodynamic turbulence at low magnetic Reynolds number. Computers $\mathscr{E}$ Fluids, vol. 50 (2011), no. 1, pp. $46-59$.

[7] C.D. Cantwell, et al. Nektar plus plus : An open-source spectral/hp element framework. Computer Physics Communications, vol. 192 (2015), pp. 205-219.

[8] G.Karniadakis and S.Sherwin. Spectral/hp Element Methods for Computational Fluid Dynamics: Second Edition. Numerical Mathematics and Scientific Computation (OUP Oxford, 2005). 
[9] G.E. Karniadakis, M. Israeli, and S.A. Orszag. High-order splitting methods for the incompressible Navier-Stokes equations. Journal of Computational Physics, vol. 97 (1991), no. 2, pp. 414-443.

[10] P.A. Davidson. Introduction to Magnetohydrodynamics (Cambridge University press, 2016), vol. 55 .

[11] C.C. Chang And T.S. Lundgren. Duct flow in magnetohydrodynamics. Zeitschrift für angewandte Mathematik und Physik ZAMP, vol. 12 (1961), no. 2, pp. 100-114.

[12] J.ShERCLIFF. Steady motion of conducting fluids in pipes under transverse magnetic fields. In Mathematical Proceedings of the Cambridge Philosophical Society (Cambridge University Press, 1953), vol. 49, pp. 136-144.

[13] M. Takashima. The stability of the modified plane Poiseuille flow in the presence of a transverse magnetic field. Fluid Dynamics Research, vol. 17 (1996), no. 6, pp. 293-310.

[14] J. Hunt. Magnetohydrodynamic flow in rectangular ducts. Journal of Fluid Mechanics, vol. 21 (1965), no. 04, pp. 577-590.

[15] J. Priede, S. Aleksandrova, And S. Molokov. Linear stability of Hunt's flow. Journal of Fluid Mechanics, vol. 649 (2010), pp. 115-134.

[16] J. Priede, S. Aleksandrova, And S. Molokov. Linear stability of magnetohydrodynamic flow in a perfectly conducting rectangular duct. Journal of Fluid Mechanics, vol. 708 (2012), pp. 111-127.

Received 01.11.2018 